Canadian University Music Review

Revue de musique des universités canadiennes

\title{
Marc-André Roberge. Ferruccio Busoni : A Bio-Bibliography. New York, Westport (Conn.) et Londres : Greenwood Press, Bio-Bibliographies in Music, $n^{\circ} 34,1991,400$ pp.
}

\section{François Tousignant}

Volume 12, numéro 1, 1992

URI : https://id.erudit.org/iderudit/1014216ar

DOI : https://doi.org/10.7202/1014216ar

Aller au sommaire du numéro

Éditeur(s)

Canadian University Music Society / Société de musique des universités canadiennes

ISSN

0710-0353 (imprimé)

2291-2436 (numérique)

Découvrir la revue

Citer ce compte rendu

Tousignant, F. (1992). Compte rendu de [Marc-André Roberge. Ferruccio Busoni : A Bio-Bibliography. New York, Westport (Conn.) et Londres : Greenwood Press, Bio-Bibliographies in Music, ${ }^{\circ}$ 34, 1991, 400 pp.] Canadian University Music Review / Revue de musique des universités canadiennes, 12(1), 130-132. https://doi.org/10.7202/1014216ar

All Rights Reserved (c Canadian University Music Society / Société de musique des universités canadiennes, 1991
Ce document est protégé par la loi sur le droit d'auteur. L’utilisation des services d'Érudit (y compris la reproduction) est assujettie à sa politique d'utilisation que vous pouvez consulter en ligne.

https://apropos.erudit.org/fr/usagers/politique-dutilisation/ 


\section{MICHEL, PIERRE}

1985 : György Ligeti,compositeurd'aujourd'hui. Paris : Minerve,Musique ouverte.

\section{NAGEL, WILLIBALD}

1917 : « Ferruccio Busoni als Aesthetiker ». Neue Musik-Zeitung, XXXVIII/15 (3 mai), 239-40; 16 (24 mai), 253-54.

\section{ROBERGE, MARC-ANDRÉ}

1991 : Ferruccio Busoni : A Bio-Bibliography. New York, Westport (Conn.) et Londres : Greenwood Press, Bio-Bibliographies in Music, $\mathbf{n}^{\circ} 34$.

\section{SCHÖNBERG, ARNOLD}

1975 : Style and Idea : Selected Writings of Arnold Schoenberg. Édité par Leonard Stein. New York: St. Martin's Press. Traduit en français sous le titre Le style et l'idée (Paris : Buchet/Chastel, 1977).

VLAD, ROMAN

1952 : «Busoni's Destiny ». The Score (décembre), 310.

1966 : « Busoni ». L'Approdo musicale, $\mathrm{n}^{\circ} 22,7-77$.

VUILLERMOZ, ÉMILE

1973 : Histoire de la musique. Édition complétée par Jacques Lonchampt. Paris : Fayard, Les grandes études historiques ( $1^{\text {re }}$ éd., 1949).

Marc-André Roberge

MARC-ANDRÉ ROBERGE. Ferruccio Busoni : A Bio-Bibliography. New York, Westport (Conn.) et Londres : Greenwood Press, Bio-Bibliographies in Music, nº 34, 1991, 400 pp.

Il est un plaisir pour tout amateur de livre d'en tenir un beau dans la main. Voilà la première et superficielle qualité qu'on note en recevant cet ouvrage de MarcAndré Roberge. Pour secondaire que cela puisse paraître, il faut quand même bien le reconnaître : le lecteur a envie de prendre ce livre, de le toucher, de le regarder et de le lire. Présentation classique, sans académisme, qualité des textures de la couverture, solidité de la reliure qui permet, avec un minimum de cassure, de laisser le livre ouvert à la page choisie - avantage non négligeable pour un ouvrage qui en est un de consultation. Aussi, grande qualité du papier, ce cher papier jauni qui ne fatigue jamais l'œil, lui laissant le loisir de se concentrer sur l'essentiel, à savoir la mine d'informations contenues en son sein.

Il s'agit ici de la trente-quatrième parution de la série «Bio-Bibliographies in Music», qui consiste davantage en un recensement des écrits du compositeur 
choisi et des écrits réalisés par des tiers sur lui qu'à une réelle biographie commentée. Initiative louable, sinon pour le grand public, du moins pour tout professeur sérieux et tout étudiant curieux. Mais venons-en directement au livre.

Dans sa courte introduction, monsieur Roberge présente un peu gauchement son sujet. Je m'explique. Dès le départ, il semble en effet pour l'auteur que l'intérêt du travail sur Busoni, et celui plus important de son œuvre, ne dépassent guère le niveau des cénacles inconditionnels (excellent recensement des diverses Sociétés Busoni) ou des cercles de recherches musicologiques universitaires. Nous aurions aimé un peu plus de passion pour le sujet en une prise de position sur la qualité et la valeur de la musique laissée par Busoni. Car, si monsieur Roberge se permet des jugements sur certains ouvrages (rendons-lui justice : il s'agit plus souvent qu'autrement d'évaluation plutôt que de jugement), il reste désespérément muet sur ce qui, au fond, est au cœur de toute activité musicale ou para-musicale : la valeur d'un compositeur et de son œuvre, bien que, dans le cas de Busoni, il faille aussi parler de l'interprète, du professeur et du théoricienvisionnaire. Busoni est, en effet, un de ces rares compositeurs-théoriciens du $\mathrm{XX}^{\mathrm{e}}$ siècle qui, à l'encontre d'autres, comme Schoenberg, Haba, Hauer, Vishnegradsky, Schaeffer..., ne mit guère en pratique (musique) le fruit de son intuition théorique.

L'esquisse biographique est sommaire, comme le voulaient probablement les contraintes de l'ouvrage. Trop, malheureusement. Si nous le déplorons, c'est qu'ici monsieur Roberge montre sa capacité à bien cerner l'essentiel; cependant, son côté schématique (et peut-être aussi sa grande intimité avec le sujet) nous prive du plaisir de 3 ou 4 pages de plus d'une lecture qui serait des plus enrichissantes, et qui nous orienterait d'autant mieux dans ce complément essentiel au livre, c'est-à-dire le désir d'une plus grande connivence avec les œuvres. Une petite coquille fait naître Ferdinand Pfohl en 1892 plutôt qu'en 1862, mais le lecteur comprendra facilement l'incohérence et l'erreur au lieu d'une mauvaise information. Entrons maintenant dans le vif du sujet - la recension de la littérature.

Il y aura sûrement des querelles de chapelles sur l'ouvrage de monsieur Roberge. Nous les lui souhaitons! Ainsi, et nous en sommes persuadé, il pourra mieux faire triompher la qualité de son travail et l'exhaustive maîtrise de son sujet. En plus d'une excellente division en rubriques des divers types d'ouvrages (œuvres, articles, livres de Busoni, critiques, lettres, travaux sur l'auteur comme : interprète, pianiste, compositeur, etc., biographies, etc.), qui allègent la recherche à l'intérieur de cet imposant colligé (on nous pardonnera le néologisme), il faut souligner surtout une recension comparative des éditions des écrits de Busoni. Ce tableau, qui peut sembler rébarbatif au premier abord, se révélera le 
plus précieux outil pour quiconque veut plonger dans la fascinante pensée musicale de Busoni. Il en va de même pour de nombreux autres aspects, notamment le catalogue des œuvres et celui des enregistrements du pianiste, qui ne manquera pas de passionner non seulement le professionnel mais également l'amateur (et nous savons combien grand est leur nombre). Nous nous permettrons une petite suggestion. Une courte section consacrée exclusivement à une liste (peut-être comparative) des biographies de Busoni, facilement consultable comme telle, aiderait fortement plusieurs lecteurs à mieux entreprendre leur connaissance de Busoni, ou de la parfaire.

Parler plus avant du cœur de ce livre serait tomber dans le dithyrambe; aussi nous contenterons-nous humblement de féliciter chaleureusement l'auteur et de recommander cet ouvrage à tout intéressé ou curieux de Busoni, ainsi que d'espérer que toute bibliothèque musicale en aura rapidement un exemplaire.

Nous n'avons qu'un regret, qu'on pourra qualifier de chauvin mais qui se justifie encore par un aveu de monsieur Roberge sur la pauvreté de l'intérêt et la piètre qualité des travaux que la francophonie a accordés à Busoni. Monsieur Roberge omet certaines traductions françaises d'articles sur Busoni (par exemple, celui de $\mathrm{H}$. Winth) et surtout, surtout, peut-être a-t-il raté l'occasion de publier, dans notre langue, cet ouvrage qui, par sa haute tenue, aurait forcé l'attention et enrichi - voire stimulé - le développement, en français, des études sur Busoni. Qu'on n'y voie pas un réflexe bassement étroit cependant. Peut-être y aura-t-il ici la répétition de l'histoire d'une certaine biographie de Mahler ... (En cela, je lance la balle dans le camp de l'édition francophone.)

En conclusion, même si le livre de monsieur Roberge peut demander un petit effort de consultation, la clarté de la méthode, l'à-propos de plusieurs commentaires ainsi que la qualité et la richesse de l'information vont en faire un ouvrage incontournable pour toute recherche sérieuse sur Busoni, et aideront plus d'un à mieux trouver (ou retrouver) quelque renseignement pertinent. Souhaitons aussi qu'il s'agisse là d'une première étape visant à une plus grande (re-) connaissance du musicien de génie qu'était Ferruccio Busoni.

François Tousignant 